\title{
Positive Lyapunov Exponents for a Class of Deterministic Potentials
}

\author{
Victor Chulaevsky ${ }^{1}$, Thomas Spencer ${ }^{2}$ \\ ' Institute for Mathematical Problems in Biology, Pushchino, Russia \\ 2 Institute for Advanced Study, Princeton, New Jersey 08540, USA
}

Received: 1 June 1993 / in revised form: 6 July 1994

Abstract: Let $V(\theta)$ be a smooth, non-constant function on the torus and let $T$ be a hyperbolic toral automorphism. Consider a discrete one dimensional Schrödinger operator $H$, whose potential at site $j$ is given by $g V_{j}=g V\left(T^{j} \theta\right)$. We prove that when $g \geqq 0$ is small and $g^{1 / 2} \leqq|E| \leqq 2-g^{1 / 2}$, the Lyapunov exponent for the cocycle generated by $H-E$ is proportional to $g^{2}$. The proof relies on a formula of Pastur and Figotin and on symbolic dynamics.

\section{Introduction}

In this paper we study the Lyapunov exponent of Schrödinger operators on a one dimensional lattice with an ergodic potential. To define an ergodic potential, let $T$ be an ergodic, measure preserving transformation of a measure space $\Theta$ with invariant measure $d v$. Let the potential at site $n$ be $V_{n}(\theta)=V\left(T^{n}(\theta)\right)$ where $V$ is a measurable function on $\Theta$. The discrete Schrödinger operator we shall study is given by

$$
(H-E) \psi_{n}=(H(\theta)-E) \psi_{n}=\psi_{n+1}+\psi_{n-1}+\left[g V_{n}(\theta)-E\right] \psi_{n} .
$$

Here $g>0$ is the coupling constant and $\psi$ is a real valued function on the integer lattice. The Lyapunov exponent defined by

$$
\gamma(E)=\lim _{N \rightarrow \infty} \frac{\log \left[\left|\psi_{N}\right|^{2}+\left|\psi_{N+1}\right|^{2}\right]}{2 N} \geqq 0
$$

is constant almost everywhere on $\Theta$ and independent of the initial values of $\psi$.

If $g>0$ and $V_{j}$ are independent random variables or $V$ comes from a Markov process, positivity of $\gamma(E)$ was proved long ago by Furstenberg [1], Virtser [2], see $[3,4]$ for a review. More generally, Simon $[5, b]$, following the original work by Kotani $[5$, a] who studied the continuous case, has shown that if the potential of the discrete one-dimensional Schrödinger operator is not determined from values in the past (the class of non-deterministic potentials) then for almost all energies $E$, the Lyapunov exponent is positive. 
For small $g$, there is a formal asymptotic expression for the Lyapunov exponent which to leading order in $g$ is given by a formula of Thouless,

$$
\gamma(E) \approx \frac{g^{2}}{2\left(4-E^{2}\right)} \rho(2 \kappa),
$$

where $\rho$ is the spectral density for the random process $V_{n}$ (i.e. the Fourier transform of the $V_{n}$ pair correlation) and $E=2 \cos \kappa$. Kappus and Wegner [6] noted that on the lattice, even when $V$ are independent, there are anomalies in the formal perturbation series in powers of $g$ for $\gamma(E)$ when $\kappa$ is a rational multiple of $2 \pi$. See [7] for a rigorous treatment of these anomalies when $V_{n}$ are independent. In the continuum, there are no anomalies provided the underlying dynamics comes from a diffusion process [8].

In this paper we obtain bounds on the Lyapunov exponent of the form (3) for a class of deterministic potentials, when the coupling constant $g$ is small and $g^{1 / 2}<|E|<2-g^{1 / 2}$. There are few rigorous bounds on Lyapunov exponents for deterministic potentials. The study of such systems is particularly natural in the context of dynamical systems. We consider a class of deterministic potentials defined by certain strongly mixing, hyperbolic dynamical systems. The main examples we have in mind are the toral automorphisms for which there is a good symbolic dynamics. In particular let $\Theta$ be the two dimensional torus and $T$ a matrix in $S L(2, \mathbb{Z})$ with a real eigenvalue strictly larger than 1 . Whenever $V$ is a smooth, non-constant function on $\Theta$ we prove that the Lyapunov exponent satisfies (3). The positivity of $\gamma(E)$ can be established using the Kotani support theorem [10] for certain values of $E$ (e.g. for almost all $E$ such that $|E| \leqq g$, see [11]) but the $g$ dependence of $\gamma(E)$ is not obtained. Note that although we assume that $T$ is uniformly hyperbolic, the cocycle defined by (1) is in general not uniformly hyperbolic. We shall also show that (3) holds for a wide class of uniformly mixing potentials such as those with Gaussian distribution whose covariance falls off faster than $|i-j|^{-2}$.

The main idea of this note comes from a very elegant analysis of Pastur and Figotin [4] who prove (3) in the independent case. What we do is to remark that independence is not crucial - the mixing which arises from toral automorphism is sufficient.

The extension of these methods to other classes of potentials with weak mixing properties, such as the skew shift, still remains to be analyzed. An interesting example is given by

$$
V_{n}=\cos \left[n \frac{(n-1)}{2} \alpha \pi+n \theta_{1}+\theta_{2}\right],
$$

where $\alpha$ is irrational and $\left(\theta_{1}, \theta_{2}\right)$ belongs to the torus. We expect that in many instances bounds of the form (3) continue to hold. It would also be very useful to extend this technique for strips or more generally for cocycles of $m$ by $m$ symplectic matrices. The main goal would be to get a lower bound on the smallest positive Lyapunov exponent in the case where $V_{j}$ are independent and $g$ is small.

The remainder of this paper is organized as follows. In the next section we present a precise formulation of our results and discuss some basic notions from symbolic dynamics. The following section is devoted to deriving the Pastur-Figotin formula which expresses the Lyapunov exponent in terms of correlation between the phase appearing in a Ricatti equation and the potential. In the final section we show 
how to estimate these correlations for small $g$ and prove Theorem 1 . In addition, we prove that (3) holds for a wide class of uniformly mixing stochastic systems.

\section{Formulation of Results}

Now we describe the class of operators for which the positivity of the Lyapunov exponents will be proven. Our aim is not to give the most general formulation of the result, but rather to keep the main ideas transparent.

Let $\mathscr{A}$ be a finite set. Without loss of generality, we can always assume that $\mathscr{A}=\{0,1, \ldots, N\}$. Consider the set $\Theta$ of all possible infinite words $\vartheta=\left(\vartheta_{n}, n \in\right.$ $\mathbb{Z}) \in \mathscr{A}^{\mathbb{Z}}$. Introduce the minimal $\sigma$-algebra $\mathscr{B}$ containing all cylinder sets

$$
C\left(i_{1}, a_{i_{1}} ; \ldots ; i_{n}, a_{n}\right)=\left\{\vartheta: \vartheta_{i_{1}}=a_{1}, \ldots, \vartheta_{l_{n}}=a_{n} ; a_{1}, \ldots, a_{n} \in \mathscr{A}\right\} .
$$

We assume the measurable space $(\Theta, \mathscr{B})$ to be equipped with a probability measure $v$, so that $(\Theta, \mathscr{B}, v)$ is a probability space. In this paper, we will always assume the measure $v$ to be invariant and ergodic under the conventional left shift transformation $T: \Theta \longrightarrow \Theta$, with

$$
(T \vartheta)_{n}=\vartheta_{n+1}
$$

and the expectation with respect to the measure $v$ will be denoted by $\mathbb{E}[\cdot]$. We assume that the measure is Markov.

Introduce a family of functionals $\operatorname{Var}_{I}, I=[a, b] \subset \mathbb{Z}$ on the space of bounded measurable functions $V$ on the measurable space $\Theta$ in the following way. First, for any interval $I \subset \mathbb{Z}$, introduce the natural projection $\pi_{I}: \mathscr{A}^{\mathbb{Z}} \longrightarrow \mathscr{A}^{I}$ by setting

$$
\pi_{I}(\vartheta)=\left(\vartheta_{i}, i \in I\right) \text {. }
$$

Then the functional $\operatorname{Var}_{I}$ is defined as follows:

$$
\operatorname{Var}_{I}(V)=\sup _{\vartheta, \vartheta^{\prime}: \pi_{I}(\vartheta)=\pi_{I}\left(\vartheta^{\prime}\right)}\left|V(\vartheta)-V\left(\vartheta^{\prime}\right)\right|
$$

Definition 1. We call a measurable function $V: \Theta \longrightarrow \mathbb{R}$ quasi-local iff for some constant $c=c(V), 0<q<1$, and for any $m, n \geqq 1$

$$
\operatorname{Var}_{[-m, n]}(V) \leqq c(V) q^{m \wedge n}, m \wedge n=\min \{m, n\} .
$$

The set of all quasi-local functions will be denoted by $\mathscr{2}(\Theta)$.

Remark. It is easy to see that if $V$ is a quasi-local function and $T$ is an automorphism of the probability space $\Theta$, then for any $t \in \mathbb{Z}, m, n \geqq 1$ we have

$$
\operatorname{Var}_{[-m, n]}\left(V\left(T^{t}(\theta)\right)\right) \leqq c(V) q^{(m+t) \wedge(n-t)}
$$

The above definitions can be easily adapted to the case of semi-infinite words $\vartheta=\left(\vartheta_{n}, n=1,2, \ldots\right) \in \mathscr{A}^{\mathbb{N}}$. In order to use unified notations, we shall write $\mathbb{X}$ for both $\mathbb{Z}$ and $\mathbb{N}$; the set of words will be denoted by $\Theta(\mathbb{X})$ or just $\Theta ; T$ will denote the left shift automorphism/endomorphism, respectively.

Now we introduce an important object related to a random process $\left(V_{n}(\vartheta)\right)$, which plays a significant role in the asymptotic analysis of the Lyapunov exponents in the weak disorder limit: the spectral density 


$$
\rho(\kappa)=\sum_{n=-\infty}^{\infty} e^{\imath \kappa n} \mathbb{E}\left[V_{0} V_{n}\right]
$$

The main results of the present paper are given by the following theorems.

Theorem 1. Let $V \in \mathscr{2}(\Theta, q)$ have zero average and let $T$ be a Markov automorphism. Consider the difference equation

$$
\left(H_{g}(\vartheta) \psi\right)(n)=\psi(n-1)+\psi(n+1)+g V\left(T^{n} \vartheta\right) \psi(n), n \in \mathbb{X}, \vartheta \in \Theta .
$$

If $g^{1 / 2}<|E|<2-g^{1 / 2}$, then for all sufficiently small $g$ the Lyapunov exponents $\gamma(E, g)$ for the solutions $\psi$ of the finite difference equations $H_{g}(x) \psi=E \psi$ is given by

$$
\begin{aligned}
\gamma(E, g) & =\frac{g^{2}}{2\left(4-E^{2}\right)}\left[\rho(2 \kappa)+\mathcal{O}\left(g \ln ^{2}(g)\left(\left|4-E^{2}\right|^{-1}+|E|^{-1}\right)\right]\right. \\
& =\frac{g^{2}}{2\left(4-E^{2}\right)}\left[\rho(2 \kappa)+\mathcal{O}\left(g^{1 / 2} \ln ^{2}(g)\right],\right.
\end{aligned}
$$

where $\cos (\kappa)=E / 2$, and $\rho(\kappa)$ is the spectral density of the random process $\left(V_{n}(\vartheta)\right)$.

Now we can give some examples of deterministic Schrödinger operators on $l^{2}(\mathbb{Z})$ and in $l^{2}(\mathbb{N})$ whose potentials belong to the classes described above.

Example 1. Hyperbolic Toral Automorphisms (Arnold's Cat Maps). Consider a matrix $M \in S L(2, \mathbb{Z})$. It naturally defines an algebraic automorphism $\tilde{M}$ of the additive group $\mathbb{T}^{2}=\mathbb{R}^{2} / \mathbb{Z}^{2}$ (the 2-torus). Let the matrix $M$ have real eigenvalues $\lambda_{+}=1 / \lambda_{-}$with $\left|\lambda_{ \pm}\right| \neq 1$. Then it is well-known (see, e.g. [9]) that if we consider the Borel $\sigma$-algebra $\mathscr{B}_{\text {Borel }}$ of subsets of $\mathbb{T}^{2}$ and the Lebesgue measure $l$ on $\mathbb{T}^{2}$, then the dynamical system $\left(\mathbb{T}^{2}, \mathscr{B}_{\text {Borel }}, l, \tilde{M}\right)$ admits a finite Markov partition and, therefore, there exists a finite set $\mathscr{A}$ and an ergodic Markov measure $v$ on the set of infinite words $\Theta=\mathscr{A}^{\mathbb{Z}}$ with the cylinder $\sigma$-algebra $\mathscr{B}$ of subsets of $\Theta$ such that the system $\left(\mathbb{T}^{2}, \mathscr{B}_{\text {Borel }}, l, \tilde{M}\right)$ is isomorphic to the left shift transformation $\tilde{T}: \Theta \longrightarrow \Theta$. Furthermore, if we consider an isomorphism $\Phi: \mathbb{T}^{2} \longrightarrow \Theta$, then the coordinates $x_{1}=x_{1}\left(\Phi^{-1}(\vartheta)\right), x_{2}=x_{2}\left(\Phi^{-1}(\vartheta)\right)$ on the torus $\mathbb{T}^{2}$ are quasilocal functions on $\Theta$. This is a direct consequence of the fact that the pre-image $\Phi^{-1}\left(C\left(-n, a_{-n} ; \ldots ; n, a_{n}\right)\right)$ of a cylinder set $C\left(-n, a_{-n} ; \ldots ; n, a_{n}\right)$ is a parallelogram of diameter $O\left(\left|\lambda_{-}\right|^{n}\right),\left|\lambda_{-}\right|<1$. Thus, if $V \in C^{1}\left(\mathbb{T}^{2}\right)$, then it corresponds to a quasi-local function $\tilde{V}(\vartheta)=V\left(\Phi^{-1}(\vartheta)\right)$ on the phase space $\Theta$ of the symbolic dynamical system $(\Theta, B, v, \tilde{T})$. These considerations lead to the following result.

Theorem 2. Consider a hyperbolic algebraic automorphism of the torus $T: \mathbb{T}^{2} \longrightarrow$ $\mathbb{T}^{2}$ and let $V \in C^{1}\left(\mathbb{T}^{2}\right)$. Then the ensemble of discrete Schrödinger operators on the space $l^{2}(\mathbb{Z})$,

$$
\left(H_{g}(x) \psi\right)(n)=\psi(n-1)+\psi(n+1)+g V\left(T^{n} x\right) \psi(n), n \in \mathbb{Z}, x \in \mathbb{T}^{2},
$$

satisfies the assumptions of Theorem 1 and, therefore, the Lyapunov exponents $\gamma(g, E)$ admit the asymptotic formula (5).

Example 2. Dyadic Circle Endomorphism. Let $\{x\}$ denote the fractional part of $x$. Consider the mapping 
Positive Lyapunov Exponents for Deterministic Potentials

$$
\begin{aligned}
T:[0,1) & \longrightarrow[0,1) \\
x & \longmapsto\{2 x\}
\end{aligned}
$$

of the interval $[0,1)$ onto itself. The binary representation of a real number $x \in[0,1)$,

$$
x=\sum_{n=1}^{\infty} \vartheta_{n} 2^{-n}, \vartheta_{n}=0,1
$$

determines an isomorphism $\Phi:[0,1) \longrightarrow\{0,1\}^{\mathbb{N}}$ of the probability spaces $([0,1)$, $\left.\mathscr{B}_{\text {Borel }}, l\right)$ and $\Theta=\{0,1\}^{\mathbf{N}}$ with the cylinder $\sigma$-algebra and the Bernoulli probability measure $v$, i.e. the probability measure such that all the variables $\vartheta_{n}$ are i.i.d. with $v\left(\left\{\vartheta_{n}=0\right\}\right)=v\left(\left\{\vartheta_{n}=1\right\}\right)=1 / 2$. Moreover, the mapping $\Phi$ defines an isomorphism of the dyadic mapping $T: x \longmapsto\{2 x\}$ and the left shift $\tilde{T}:\left(\vartheta_{1}, \vartheta_{2}, \ldots\right) \longmapsto\left(\vartheta_{2}, \vartheta_{3}, \ldots\right)$ on $\Theta$. Again, the coordinate $x=x\left(\Phi^{-1}(\vartheta)\right)$ is a quasi-local function on $\Theta$, since not only the probability, but also the diameter of a cylinder set $\left\{\vartheta_{1}=a_{1}, \ldots \vartheta_{n}=a_{n}\right\}$ equals $2^{-n}$. So, Theorem 1 implies the following statement.

Theorem 3. Consider the dyadic transformation $T:[0,1) \longrightarrow[0,1)$ and let $V$ be a smooth periodic function on $([0,1])$. Then the ensemble of discrete Schrödinger operators on the space $l^{2}(\mathbb{N})$,

$$
\left(H_{g}(x) \psi\right)(n)=\psi(n-1)+\psi(n+1)+g V\left(T^{n} x\right) \psi(n), n \in \mathbb{N}, x \in \mathbb{T}^{2},
$$

with a self-adjoint (e.g. Dirichlet) boundary condition at $n=0$ satisfies assumptions of Theorem 1 and, therefore, the Lyapunov exponents $\gamma(g, E)$ admit the asymptotic formula (5).

\section{Remarks.}

1. In most cases $\rho(\kappa)>0$, which yields a quadratic bound from below for the Lyapunov exponents. For example, all pair correlations $\mathbb{E}\left[v_{0} v_{n}\right], n \neq 0$, may van$i s h$, as it is the case for $V_{n}(\vartheta)=\cos \left(2 \pi 2^{n} \vartheta\right), n \in \mathbb{N}$. If $\mathbb{E}\left[v_{0} v_{n}\right]=\delta_{n 0}$, then $\rho(\kappa) \equiv 1$.

2. It can be seen from our proof that the leading term of the above asymptotic formulas is valid for all $g>0, E \in(-2,2) \backslash\{0\}$ satisfying

$$
\sqrt{4-E^{2}} \geqq g^{\alpha}>0, \quad|E| \geqq g^{\alpha}>0,
$$

for some positive number $\alpha<1$. However, in the upper bound for the remainder, the factor of $g^{5 / 2} \ln ^{2} g^{-1}$ should be replaced with $g^{3-\alpha} \ln ^{2} g^{-1}$.

3. We do not consider the case $|E|>2$, since for any such $E$ there exists $g_{o}(E)>0$ so that if $|g|<g_{o}(E)$, then $E$ does not belong to the spectrum of $H_{g}$.

4. The condition of smoothness of the function $V: \Theta \longrightarrow \mathbb{R}$ can be relaxed to the Hölder continuity, $\left|V(\vartheta)-V\left(\vartheta^{\prime}\right)\right| \leqq A\left|\vartheta-\vartheta^{\prime}\right|^{b}, 0<b \leqq 1$, since this does not violate the quasi-locality condition, but only affects the exponent of decay of the tail-dependence of the functionals $\operatorname{Var}_{I}(V)$. 


\section{The dynamic formalism for the Lyapunov exponents}

In this section we derive an expression for the Lyapunov exponent by going to suitable polar coordinates. Our derivation follows [4]. We use the matrix formulation of the equation, $H(\theta) \psi=E \psi$, which may be written as

$$
\left(\begin{array}{c}
\psi_{n+1} \\
\psi_{n}
\end{array}\right)=M_{n}\left(\begin{array}{c}
\psi_{n} \\
\psi_{n-1}
\end{array}\right) \equiv\left(\begin{array}{cc}
E-g v_{n} & -1 \\
1 & 0
\end{array}\right)\left(\begin{array}{c}
\psi_{n} \\
\psi_{n-1}
\end{array}\right) \text {. }
$$

For $|E|<2$, set $E=2 \cos \kappa$ and let

$$
\begin{aligned}
\left(y_{n}, y_{n-1}\right) & =\left(\psi_{n}-\cos \kappa \psi_{n-1}, \sin \kappa \psi_{n-1}\right) \\
& \equiv \rho_{n}\left(\cos \varphi_{n}, \sin \varphi_{n}\right) .
\end{aligned}
$$

The advantage of the $y$ variables is that for $g=0$ they simply rotate by angle $\kappa$. For $g \neq 0$ we have,

$$
\begin{aligned}
\left(\begin{array}{c}
y_{n+1} \\
y_{n}
\end{array}\right)= & {\left[\begin{array}{cc}
\cos \kappa & -\sin \kappa \\
\sin \kappa & \cos \kappa
\end{array}\right]\left(\begin{array}{c}
y_{n} \\
y_{n-1}
\end{array}\right) } \\
& -\frac{g v_{n}}{\sin \kappa}\left[\begin{array}{cc}
\sin \kappa & \cos \kappa \\
0 & 0
\end{array}\right]\left(\begin{array}{c}
y_{n} \\
y_{n-1}
\end{array}\right)
\end{aligned}
$$

In polar coordinates we find the relation

$$
\rho_{n+1}\left(\begin{array}{c}
\cos \varphi_{n+1} \\
\sin \varphi_{n}
\end{array}\right)=\rho_{n}\left(\begin{array}{c}
\cos \left(\bar{\varphi}_{n}\right)+g V_{n} \sin \left(\bar{\varphi}_{n}\right) \\
\sin \left(\bar{\varphi}_{n}\right)
\end{array}\right),
$$

where we have set

$$
V_{n}=-\frac{v_{n}}{\sin \kappa} \text { and } \bar{\varphi}=\varphi+\kappa
$$

By taking the ratio of the upper and lower entries of (7) and the norm of (7) we see that

$$
\cot \varphi_{n+1}=\cot \bar{\varphi}_{n}+g V_{n}
$$

and

$$
\rho_{n+1}^{2}=\rho_{n}^{2}\left(1+g V_{n} \sin (2 \bar{\varphi})+g^{2} V_{n}^{2} \sin ^{2} \bar{\varphi}\right) .
$$

Since the Lyapunov exponent is independent of coordinate frame, we see that by taking the $\log$ of the right-hand side of $(8 \mathrm{~b})$ that

$$
\begin{aligned}
\gamma(E)= & \lim _{n \rightarrow \infty}(\pi N)^{-1} \int_{0}^{\pi} d \varphi_{0} \mathbb{E} \ln \left[\rho_{N}\right] \\
= & g^{2} \frac{E\left(v_{0}^{2}\right)}{8}+\frac{1}{2 \pi N} \sum_{n=1}^{N} \int_{0}^{\pi} d \varphi_{0} \mathbb{E}\left[g V_{n} \sin \left(2 \bar{\varphi}_{n}\right)-\frac{g^{2} V_{n}^{2}}{2} \cos (2 \bar{\varphi})\right. \\
& \left.+\frac{1}{4} g^{2} V_{n}^{2} \cos \left(4 \bar{\varphi}_{n}\right)\right]+\mathcal{O}\left(g^{3} / \sin ^{3} \kappa\right) .
\end{aligned}
$$

The above representation for the Lyapunov exponent shows that $\gamma(E)>0$ for sufficiently small $g$ if all the correlations $\mathbb{E}[V \sin 2 \bar{\varphi}], \mathbb{E}\left[V^{2} \sin 2 \bar{\varphi}\right], \mathbb{E}\left[V^{2} \cos 4 \bar{\varphi}\right]$ are small enough for small $g$. 
In order to estimate the " $V-\varphi$ " correlations, introduce the variable $\zeta_{n}=$ $\exp \left(2 i \varphi_{n}\right)$. Then we can rewrite (8) as the evolution equation for $\zeta_{n}$ driven by the random process $V_{n}$ :

$$
\zeta_{n+1}=\mu \zeta_{n}+\frac{i g}{2} V_{n} \frac{\left(\mu \zeta_{n}-1\right)^{2}}{1-\frac{i g}{2} V_{n}\left(\mu \zeta_{n}-1\right)}
$$

with $\mu=e^{2 \imath \kappa}$. So, our problem can be reduced to estimation of the " $V-\zeta$ ", " $V^{2}-\zeta$ ", and " $V^{2}-\zeta^{2}$ " correlations, since $V$ is real-valued and $2 i \sin 2 \varphi=\zeta-$ $\zeta^{*}, 2 i \sin 4 \varphi=\zeta^{2}-\left(\zeta^{*}\right)^{2}$, where $\zeta^{*}$ stands for the complex conjugate of $\zeta$. So, in the rest of this paper we will prove those asymptotic upper bounds for the " $V-\zeta$ ", " $V-\zeta^{2}$ " correlations for a wide class of potentials $V_{n}$ including those generated by chaotic dynamical systems on finite-dimensional manifolds.

Before proceeding to the next section we estimate the average of $\zeta$ and $\zeta^{2}$ following [4]. Also, for notational simplicity, we shall denote from now on all three kinds of averages which appear at the r.h.s. of the key representation (9) as $<\cdot>$, e.g.

$$
\left\langle\zeta_{t}\right\rangle=\lim _{N \rightarrow \infty}(\pi N)^{-1} \sum_{n=0}^{N-1} \int_{0}^{\pi} d \phi_{0} \mathbb{E}\left[\zeta_{n}\right] .
$$

Note that $\left\langle\zeta_{t}\right\rangle$ is independent of $t$; however, averages like $\left\langle\zeta_{t} V_{t+s}\right\rangle$ depend upon $s$.

\section{Lemma 1.}

(i) For $|E|<2$,

$$
\left\langle\zeta_{t}\right\rangle=O\left(\frac{g}{\sin ^{2} \kappa}\right)
$$

(ii) For $E \neq 0$,

$$
\left\langle\zeta_{t}^{2}\right\rangle=O\left(\frac{g}{\sin \kappa \sin 2 \kappa}\right)
$$

Proof. From the evolution equation (10) for $\zeta_{t}$ we get

$$
\left\langle\zeta_{t}\right\rangle=e^{2 i \kappa}\left\langle\zeta_{t-1}\right\rangle+O(g / \sin \kappa)=e^{2 i \kappa}\left\langle\zeta_{t}\right\rangle+O(g / \sin \kappa),
$$

so that

$$
\left\langle\zeta_{t}\right\rangle\left(1-e^{2 i \kappa}\right)=O(g / \sin \kappa)
$$

and similarly

$$
\left\langle\zeta_{t}^{2}\right\rangle\left(1-e^{4 l \kappa}\right)=O(g / \sin \kappa),
$$

which leads to the required estimates.

\section{Upper Bounds for $V-\zeta$ Correlation}

Consider a domain $D=\{|u| \leqq 1,|\zeta| \leqq 1\} \subset \mathbb{C}^{2}$ and define on $D$ the following function:

$$
F(\zeta, u)=\mu \zeta+i g u \frac{(\mu \zeta-1)^{2}}{1-i g u(\mu \zeta-1)},
$$

where $|\mu|=1$ and $|g| \leqq 1 / 4$, so that the denominator $1-i g u(\mu \zeta-1)$ does not vanish in $D$ and $F$ is holomorphic in a neighborhood of the closure of $D$. The 
latter property of $F$ implies immediately that $F$ is uniformly Lipshitz continuous in $D$. The following simple statement gives more precise information about continuity of $F$.

Lemma 2. For any $\left(\zeta_{1}, u_{1}\right),\left(\zeta_{2}, u_{2}\right) \in D$,

$$
\left|F\left(\zeta_{1}, u_{1}\right)-F\left(\zeta_{2}, u_{2}\right)\right| \leqq(1+O(g))\left|\zeta_{1}-\zeta_{2}\right|+O(g)\left|u_{1}-u_{2}\right| \text {. }
$$

Proof. A direct calculation shows that

$$
|\partial F / \partial \zeta| \leqq|\mu|+O(g)=1+O(g), \quad|\partial F / \partial u| \leqq O(g)+O\left(g^{2}\right),
$$

uniformly in $D$, yielding the desired inequalities.

Let $V: \Theta \longrightarrow \mathbb{R}$ be a quasi-local function and $\left\{\zeta_{t}\right\}$ be the solution to the evolution equation (10), driven by the random process $\left\{V_{t}\right\}$. Note that $V_{n}=v_{n} / \sin \kappa$, where $v$ is as in the previous section. As a result, the $V$ defined in the first two sections differs by a factor of $\sin \kappa$ from the $V$ of this and the previous section.

Lemma 3. For any $t \geqq 0$ and any interval $I$ which contains the points $t, t+1$,

$$
\operatorname{Var}_{I}\left(\zeta_{t+1}\right) \leqq(1+O(g)) \operatorname{Var}_{I}\left(\zeta_{t}\right)+O(g) \operatorname{Var}_{I}\left(V_{t}\right)
$$

Proof. Apply Lemma 2 and (10).

Our main lemma can be stated as follows.

Lemma 4. For sufficiently small $g$, for any $T \geqq 2$ and any $t \geqq 1$,

$$
\operatorname{Var}_{(-\infty, t+T]}\left(\zeta_{t}\right) \leqq O\left(\frac{q^{T} g}{\sin \kappa}\right)
$$

Proof. Using Lemma 3, we can write

$$
\operatorname{Var}_{[-\infty, t+T]}\left(\zeta_{m}\right) \leqq(1+C|g|) \operatorname{Var}_{[-\infty, t+T]}\left(\zeta_{m-1}\right)+C|g| \operatorname{Var}_{[-\infty, t+T]}\left(V_{m-1}\right),
$$

for some constant $C$. Therefore,

$$
\begin{aligned}
\operatorname{Var}_{[-\infty, t+T]}\left(\zeta_{t}\right) \leqq & C|g| \sum_{s=1}^{t}(1+C|g|)^{s-1} \operatorname{Var}_{[-\infty, t+T]}\left(V_{t-s}\right) \\
& +(1+C|g|)^{t} \operatorname{Var}_{[-\infty, t+T]}\left(\zeta_{0}\right)
\end{aligned}
$$

Notice that $\operatorname{Var}_{[-\infty, t+T]}\left(\zeta_{0}\right)=\operatorname{Var}_{[-\infty, t+T]}($ const $)=0$. Furthermore, since $V$ is quasi-local,

$$
\operatorname{Var}_{[-\infty, n+r]}\left(V_{n}\right) \leqq c_{1}(V) q^{r}
$$

After we substitute (13) into the r.h.s. of (12) we have

$$
\begin{aligned}
\operatorname{Var}_{[-\infty, t+T]}\left(\zeta_{t}\right) & \leqq C|g| c(V) \sum_{s=1}^{t}(1+C|g|)^{s} q^{T+s} \\
& \leqq O\left(\frac{g}{\sin \kappa}\right) q^{T} \sum_{s=1}^{\infty} q^{s}(1+C|g|)^{s}=O\left(\frac{q^{T} g}{\sin \kappa}\right)
\end{aligned}
$$


Now we shall estimate the " $V-\varphi$ " correlations that appear in (9). Let $\bar{\zeta}=\mu \zeta$.

Lemma 5. For small $g$,

$$
\left\langle\bar{\zeta}_{t} V_{t}\right\rangle=\frac{i g}{4}\left(\rho_{V}(2 \kappa)-\left\langle V_{0}^{2}\right\rangle\right)+O\left(\frac{g^{2} \ln ^{2} g}{\sin ^{3} \kappa \sin 2 \kappa}\right) .
$$

Proof. Using the evolution equation (10), we see that

$$
\begin{aligned}
\left\langle\bar{\zeta}_{t+T} V_{t+T}\right\rangle= & \mu^{T}\left\langle\bar{\zeta}_{t} V_{t+T}\right\rangle+\frac{i g}{2} \sum_{s=0}^{T-1} \mu^{T-s}\left\langle V_{t+s} V_{t+T}\right\rangle \\
& +\frac{i g}{2} \sum_{s=0}^{T-1} \mu^{(T+s)}\left\langle\bar{\zeta}_{t}^{2} V_{t+s} V_{t+T}\right\rangle-i g \mu^{T} \sum_{s=0}^{T-1}\left\langle\overline{\zeta \zeta}_{t} V_{t+s} V_{t+T}\right\rangle+O\left(\frac{T^{2} g^{2}}{\sin ^{2} \kappa}\right)
\end{aligned}
$$

We shall prove that the terms on the right side of (14) containing factors of $\zeta$ approximately factor. Using Lemma 1 and the fact that $V$ has zero average we shall see these terms are relatively small. More precisely set $T=A[\ln g / \ln q]$, where [ - ] stands for the integer part of a real number, and $A \geqq 4$. Define functions $\Theta_{t}=\Theta_{t}(\vartheta)$ and $\bar{\Theta}_{t}$ as follows:

$$
\left(\Theta_{t}\right)_{n}=\left(\Theta_{t}(\vartheta)\right)_{n}= \begin{cases}\vartheta_{n}, & \text { if }|n-t| \leqq T / 4 \\ 0, & \text { otherwise }\end{cases}
$$

and

$$
\left(\bar{\Theta}_{t}\right)_{n}=\left(\bar{\Theta}_{t}(\vartheta)\right)_{n}= \begin{cases}\vartheta_{n}, & \text { if } n \leqq t+T / 4 \\ 0, & \text { otherwise. }\end{cases}
$$

Then we can represent $\zeta_{t}$ and $V_{t+s}$ as

$$
\begin{gathered}
\zeta_{t}=\zeta_{t}\left(\bar{\Theta}_{t}\right)+\left(\zeta_{t}-\zeta_{t}\left(\bar{\Theta}_{t}\right)\right)=\zeta_{t}\left(\bar{\Theta}_{t}\right)+O\left(\frac{g q^{T / 2}}{\sin \kappa}\right) \\
V_{t+s}=V_{t+s}\left(\Theta_{t+s}\right)+\left(V_{t+s}-V_{t+s}\left(\Theta_{t+s}\right)\right)=V_{t+s}\left(\Theta_{t+s}\right)+O\left(\frac{q^{T / 2}}{\sin \kappa}\right),
\end{gathered}
$$

by virtue of Lemma 4 and assumption of quasi-locality of $V_{t}$. So, the first term on the r.h.s. of (14) is bounded by the strong mixing of the Markov system

$$
\begin{aligned}
& \left\langle\left(\zeta_{t}\left(\bar{\Theta}_{t}(\vartheta)\right)+O\left(\frac{g q^{T / 2}}{\sin \kappa}\right)\right)\left(V_{t+T}\left(\Theta_{t+T}(\vartheta)\right)+O\left(\frac{q^{T / 2}}{\sin \kappa}\right)\right)\right\rangle \\
& =\left\langle\zeta_{t}\left(\bar{\Theta}_{t+T}(\vartheta)\right)\right\rangle\left\langle V_{t+T}\left(\Theta_{t+T}(\vartheta)\right)\right\rangle+O\left(\frac{g^{A / 2}}{\sin \kappa}\right)=O\left(\frac{g^{A / 2}}{\sin \kappa}\right),
\end{aligned}
$$

since $\langle V\rangle=0$ and $|g / \sin \kappa| \leqq 1$. The second term simply gives rise to

$$
\begin{aligned}
\frac{i g}{2} \sum_{n=1}^{T-1} e^{2 i \kappa n}\left\langle V_{0} V_{n}\right\rangle & =\frac{i g}{4} \sum_{n=-\infty}^{\infty} e^{2 i \kappa n}\left\langle V_{0} V_{n}\right\rangle-\frac{i g}{4}\left\langle V_{0}^{2}\right\rangle+O\left(\frac{q^{T}}{\sin ^{2} \kappa}\right) \\
& =\frac{i g}{4}\left(\rho_{V}(2 \kappa)-\left\langle V_{0}^{2}\right\rangle\right)+O\left(\frac{g^{A}}{\sin ^{2} \kappa}\right),
\end{aligned}
$$


$\rho_{V}(\kappa)$ being the Fourier transform of the correlation function $\left\langle V_{0} V_{n}\right\rangle$. The third and the fourth terms are quite similar, so we focus on the fourth one given by the sum

$$
g \sum_{s=0}^{T-1}\left\langle\zeta_{t} V_{t+s} V_{t+T}\right\rangle
$$

Terms in (15) with $s \leqq T / 2$ are bounded by

$$
\left\langle\zeta_{t}\left(\bar{\Theta}_{t}\right) V_{t+s}\left(\Theta_{t+s}\right)\right\rangle\left\langle V_{t+T}\left(\Theta_{t+T}\right)\right\rangle+O\left(\frac{q^{T / 2}}{\sin ^{4} \kappa}\right)=O\left(\frac{g^{A / 2}}{\sin ^{4} \kappa}\right) .
$$

Terms in (15) with $T / 2 \leqq s$ can be estimated as follows. By (11a) $g\left\langle\zeta_{t} V_{t+s} V_{t+T}\right\rangle$ is bounded by

$$
\begin{aligned}
& g\left\langle\zeta_{t} V_{t+s} V_{t+T}\right\rangle \\
& =g\left\langle\zeta_{t}\left(\Theta_{t}\right)\right\rangle\left\langle V_{t+s}\left(\Theta_{t+s}\right) V_{t+T}\left(\Theta_{t+T}\right)\right\rangle+O\left(\frac{q^{T / 2}}{\sin ^{4} \kappa}\right)=O\left(\frac{g^{2}}{\sin ^{4} \kappa}\right) .
\end{aligned}
$$

In a similar fashion, the third term in (14) is bounded by

$$
O\left(\frac{g^{2}}{\sin ^{3} \kappa \sin 2 \kappa}\right) \text {. }
$$

Combining all the above estimates, the lemma follows.

Corollary. For small $g$,

$$
\begin{aligned}
g\left\langle V_{t} \sin 2 \varphi_{t}\right\rangle & =\frac{g^{2}}{4}\left(\rho_{V}(2 \kappa)-\left\langle V_{0}^{2}\right\rangle\right)+O\left(\frac{g^{3} \ln ^{2} g}{\sin ^{3} \kappa \sin 2 \kappa}\right) \\
& =\frac{g^{2}}{4 \sin ^{2} \kappa}\left(\rho_{v}(2 \kappa)-\left\langle v_{0}^{2}\right\rangle\right)+O\left(\frac{g^{3} \ln ^{2} g}{\sin ^{3} \kappa \sin 2 \kappa}\right)
\end{aligned}
$$

Lemma 6. For small $g$

$$
\left\langle\zeta_{t+T} g^{2} V_{t+T}^{2}\right\rangle=O\left(\frac{g^{3} \ln ^{2} g}{\sin ^{3} \kappa \sin 2 \kappa}\right)
$$

and

$$
\left\langle\zeta_{t+T}^{2} g^{2} V_{t+T}^{2}\right\rangle=O\left(\frac{g^{3} \ln ^{2} g}{\sin ^{2} \kappa \sin 2 \kappa}\right)
$$

The upper bounds for the correlations (17) and (18) can be proven in a similar way with the help of Lemma 4 combined with Lemma 1.

Proof of Theorem 1. Combining the representation (9) for the Lyapunov exponents with the asymptotic formula (16) for the correlation $g\left\langle V_{t} \sin 2 \varphi_{t}\right\rangle$ and with the upper bounds for other correlations in (9) given by Lemmas 5-6, we have 


$$
\begin{aligned}
\gamma(E) & =\frac{g^{2}}{8} \mathbb{E}\left[V_{0}^{2}\right]+\frac{g}{2}\left\langle V_{t} \sin 2 \varphi_{t}\right\rangle+O\left(\frac{g^{3} \ln ^{2} g}{\sin ^{3} \kappa \sin 2 \kappa}\right) \\
& =\frac{g^{2}}{8 \sin ^{2} \kappa}\left\langle v_{0}^{2}\right\rangle+\frac{g^{2}}{8 \sin ^{2} \kappa}\left(\rho(2 \kappa)-\left\langle v_{0}^{2}\right\rangle+O\left(\frac{g \ln ^{2} g}{\sin \kappa \sin 2 \kappa}\right)\right) \\
& =\frac{g^{2}}{2\left(4-E^{2}\right)}\left(\rho(2 \kappa)+O\left(\frac{g \ln ^{2} g}{\sin \kappa \sin 2 \kappa}\right)\right) .
\end{aligned}
$$

Since $E=2 \cos \kappa$ and $g^{1 / 2} \leqq|E| \leqq 2-g^{1 / 2}$ we have

$$
O\left(\frac{g \ln ^{2} g}{\sin \kappa \sin 2 \kappa}\right) \leqq g^{1 / 2} \ln ^{2} g
$$

This completes the proof.

As it was mentioned before, Theorems 2 and 3 follow from Theorem 1.

Remark. It is worth mentioning that the present approach, of Pastur and Figotin [4], allows us to prove in a much simpler way the asymptotic formula for the Lyapunov exponents for ergodic operators $H_{g}$ with the stochastic potentials $V_{t}$ satisfying the Rosenblatt strong mixing condition. Namely, assume that for $n>0, F_{-n}(V)$ is any random variable determined by the values $\left\{V_{-n-m}, m \geqq 0\right\}$ of the random process $V_{t}$, and $G_{n}(V)$ is any random variable determined by $\left\{V_{n+m}, m \geqq 0\right\}$. Suppose we have

$$
\left|\left\langle F_{-n}(V) G_{m}(V)\right\rangle-\left\langle F_{-n}(V)\right\rangle\left\langle G_{m}(V)\right\rangle\right| \leqq C|n+m|^{-\alpha}
$$

with $\alpha>1$, then the decoupling of the correlations $\left\langle V_{t+T} \zeta_{t}\right\rangle,\left\langle V_{t+T}^{2} \zeta_{t}\right\rangle,\left\langle V_{t+T}^{2} \zeta_{t}^{2}\right\rangle$ for $T \simeq g^{-\beta}, 0<\beta<1 / 2$, follow directly from the above strong mixing condition, since $\zeta_{t}$ is completely determined by $\left\{V_{s}, s<t\right\}$. So, if $\beta<1 / 2$, and $\alpha>1$, then we get the required estimates for the " $V-\zeta$ " correlations. Indeed, as in Lemma 5, (14) implies that for $T=g^{-\beta} \beta=\left(\frac{2}{2+\alpha}\right)$,

$$
\left\langle\zeta_{t+T} V_{t+T}\right\rangle=\frac{i g}{4}\left[\rho_{V}(2 \kappa)-V_{0}^{2}+\mathcal{O}\left(\frac{g^{(\alpha-1)}+g^{\frac{2 \gamma}{2 \gamma+1}}}{\sin ^{3} \kappa \sin 2 \kappa}\right)\right] .
$$

In a similar way, one can show the $V^{2}-\zeta$ and $V^{2}-\zeta^{2}$ correlations are relatively small.

Acknowledgement. We wish to thank the referee for carefully reading an earlier version of this paper and suggesting a number of corrections and improvements.

\section{References}

1. Furstenberg, H.: Noncommuting random products. Trans. Am. Math. Soc. 108, 377-428 (1963)

2. Virtser, A.D.: On products of random matrices and operators. Prob. Theory Appl. 24, 367-377 (1979)

3. Carmona, R., Lacroix, J.: Spectral Theory of Random Schrödinger Operators Boston Bosel: Birkhäuser, 1990 
4. Pastur, L., Figotin, A.: Spectra of Random and Almost- Periodic Operators. Berlin, Heidelberg, New York: Springer, 1992

5. (a) Kotani, S.: Lyapunov indices determine absolutely continuous spectrum of stationary onedimensional Schrödinger operators. In: Proc. Taneguchi Intern. Symp. on Stochastic Analysis. Katadata and Kyoto, K.Ito (ed.), Amsterdam, North Holland, 1982, pp. 225-247;

(b) Simon, B.: Kotani theory for one-dimensional stochastic Jacobi matrices. Commun. Math. Phys. 89, 227 (1983)

6. Kappus, M., Wegner, F.: Anomaly in the Band Centre of the One-Dimensional Anderson Model. Z. Phys. B45, 15-21 (1981)

7. Campanino, M., Klein, A.: Anomalies in the One-Dimensional Anderson Model. Commun. Math. Phys. 130, 441-456 (1990)

8. Arnold, L., Papanicolaou, G., Wishtutz, V.: Asymptotic analysis of the Lyapunov exponent and rotation number of a random oscillator and applications. SIAM J. Appl. Math. 46, 427-450 (1986)

9. Kornfeld, I.P., Fomin, S.V., Sinai, Ya.G.: Ergodic theory. Berlin, Heidelberg, New York: Springer 1982

10. Kotani, S.: Support theorems for random Schrödinger operators; Commun. Math. Phys. 97, 443-452 (1985)

11. Spencer, T.: Ergodic Schrödinger operators. In: Analysis, et cetera, Rabinowitz, P., Zehnder, E. (eds.), New York: Academic Press, (1990)

Communicated By B. Simon 